\title{
Knowledge-Managing Sustainable Energy Schemes - An Innovative Approach
}

\author{
Anthony Ayoola
}

\begin{abstract}
Sustainable energy management systems continue to evolve, adding layers of complexity and functionality. The paper presents an innovative approach that deals with the application of complex systems formalism and contemporary knowledge management techniques for the purposes of assuring more effective delivery of sustainable energy schemes.

Knowledge-Managed Sustainable Energy (KMSE) systems leverage energy knowledge assets optimally, and with greater flexibility, to enhance energy management performance. The KMSE scheme developed adopts a multi-factorial approach for sustainable energy-related knowledge acquisition, aggregation and diffusion, within managed environments.

The paper presents a structured framework for developing effective KMSE systems, and discusses their applicability to a range of renewable energy management projects, with the ultimate aim of fostering continuous energy management performance improvement and sustainability.
\end{abstract}

Index Terms-Energy Kiviats, energy management knowledge, interaction circles, knowledge diffusion, knowledge externalization, knowledge management, sustainable energy.

\section{INTRODUCTION AND BACKGROUND}

Sustainable energy management systems attempt to provide long-term cost-effective management of energy provision or use. Our study focuses on the Development and Management of Sustainable Energy Schemes.

We have developed a scalable scheme applicable to

1) Sub-Units (e.g. Departments)

2) Units (e.g. Organizations)

3) Super-units (e.g. Cities \& Regions)

Standard Energy Management approaches, such as those based on international energy management standards e.g. ISO 50001/BS EN, including those purporting to utilize knowledge-based schemes [1]-[6], are typically linear, with feedback loops often used to regulate and fine-tune the process (as shown in Fig. 1 overleaf):

A new energy management approach - termed the 'Knowledge-Managed Sustainable Energy (KMSE)' technique has been developed, with the attributes shown in Fig. 2.

KMSE adopts a complex systems approach to analysing, designing, monitoring and controlling energy management schemes [7]. KMSE ensures that energy knowledge assets are appropriately developed, aggregated and diffused within the

Manuscript received November 17, 2013; revised May 27, 2014. This work was supported by Hanta Associates, Consultancy and Research Division, United Kingdom

Anthony Ayoola is with the School of Business \& Management, American University of Ras Al Khaimah, Ras Al Khaimah, United Arab Emirates (e-mail: Anthony.Ayoola@aurak.ae). unit, sub-unit or super-unit.

The scheme works with two distinct phases - Hard and Soft KMSE Phases.

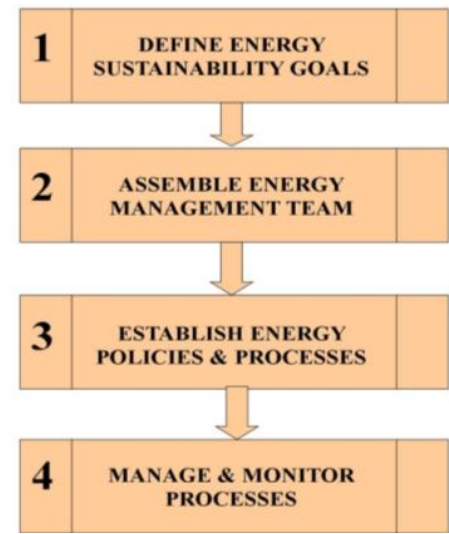

Fig. 1. Standard linear energy management workflow.

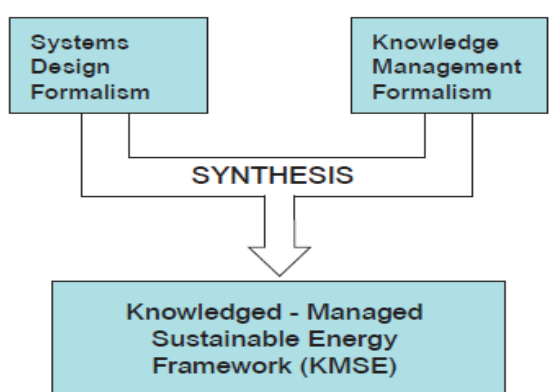

Fig. 2. New knowledge-based energy management approach.

\section{II. "HARD” KMSE PHASE}

The Hard KMSE phase involves systemic analysis of energy systems and processes.

It features standardised elements based on formal technical analyses of

1) Energy sources, sinks \& options for these

2) Energy conversion efficiencies

The phase requires determination of feasibility of the various energy provision and usage options available essentially a consideration of technical, operational \& financial feasibility of these items.

\section{III. “SOFT" KMSE PHASE}

The Soft KMSE phases manage tacit and explicit knowledge sources within the sub-unit, unit and/or super-unit. Soft KMSE is predicated on key Knowledge management (KM) phases which are in turn based on Socialization, Externalization, Combination (Group-learning and Dissemination) and Internalization KM activities. 
Approaches of this nature are termed SECI-based Systemic Models [8]-[13].

Whilst generic KM approaches and models are useful, they have limited utility unless they are adapted for managing sustainable energy schemes. As such, we have used SECI-based models to formulate a more relevant and robust framework to underpin the proposed KMSE technique. This adaptation is shown in Fig. 3.

\section{N - Phase KMSE Scheme}

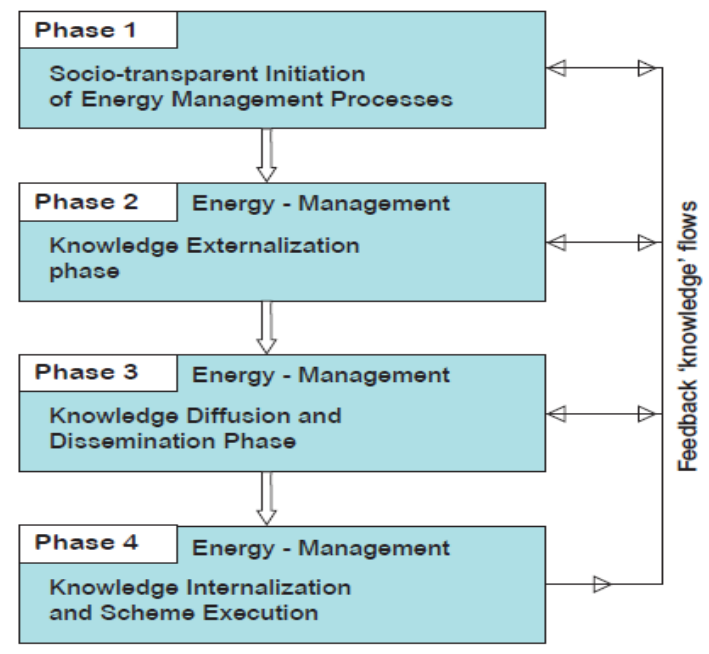

Fig. 3. Multi-phased KMSE workflow.

Elaborating further on the Soft KMSE phases.

\section{Phase 1: Initiation OF ENERGy MANAGEMENT PROCESSES}

Phase 1 entails identification of energy stakeholders and energy assets within the organization. It also requires the specification of usable metrics that conform to 'SMART' criteria. That is, they have to be specific, measurable, achievable, relevant and time-bound.

SMART KPIs would typically fall into categories relating to:

1) Cost-benefit metrics

2) Return-On-Investment/Payback metrics

3) Energy conversion efficiency metrics

4) Carbon reduction metrics

5) Environmental compliance metrics.

Ideally, the KPIs assembled within these categories should cross-reference industry standard benchmarks. The ensemble of metrics or KPIs so derived, notionally form the basis for "Energy Intelligence" functionality within the KMSE scheme.

\section{PhASE 2: KNOWLEDGE EXTERNALIZATION PHASE}

The previous phase effectively collates foundational energy management knowledge. A significant component of this is tacit or implicit energy management knowledge.

That is, many strands of the energy management know-how are informal and known primarily to specific individuals and/or areas of the organization.

In phase 2 we finalize the collation of underpinning energy management tacit or implicit energy management knowledge.
Within this phase we formalize and render explicit all the main tacit knowledge strands pertaining to energy management. A key requirement within this phase is the creation of Energy Knowledge Interaction Circles (EKICs) - Small groupings of key personnel involved with energy management within the unit (termed the Socialization Sub-phase) [14]-[19].

As a final developmental sub-phase for the KMSE scheme, it will be necessary to create energy management and usage models that can be used to model energy usage over reasonably extended timelines. The main thrust of the models-assembly sub phase is to allow continuous verification of metrics based on a comparison of actual and modelled (projected) energy management performance. The assessed variances can serve as triggers for tuning of the KMSE scheme.

A crucial aspect of the sub-phase will be to establish 'action-thresholds'. These are effectively variance values that trigger corrective or tuning action for the KMSE. It would be helpful to collate complementary or related energy management/usage models into the clearly defined high-level frameworks. A fully evolved, mature, KMSE could have a number of such frameworks embedded within the scheme, used to govern or regulate different aspects of the energy management scheme. To support phases 1 \& 2, a cross-mapping matrix (CMM) relating energy management objectives and KPI/metrics categories can be used - a sample CMM is shown in Table I below:

TABLE I: SAMPle TeMPlate ENERGy MANAGEMENT OBJECTIVES - KPI CROSS-MAPPING MATRIX

\begin{tabular}{|c|c|c|c|c|}
\hline KPI/Metric & \multicolumn{4}{|c|}{ Energy Management Objectives } \\
\hline & Objective 1 & Objective 2 & Objective 3 & Objective 4 \\
\hline $\begin{array}{l}\text { Financial } \\
\text { Metrics }\end{array}$ & $\begin{array}{l}\text { KPI - 1 } \\
\text { Asset - } 1\end{array}$ & & & \\
\hline $\begin{array}{l}\text { Energy } \\
\text { use/conversio } \\
\mathrm{n} \quad \text { metrics } \\
\text { efficiency }\end{array}$ & & & & \\
\hline $\begin{array}{l}\text { Carbon } \\
\text { reduction } \\
\text { metrics }\end{array}$ & & & & \\
\hline
\end{tabular}

The row-column intersections or cells of the CMM serve to specify particular metric items and energy management knowledge assets that are applicable for the KMSE.

Phase 2 can be succinctly summarized by the diagram shown in Fig. 4.

\section{Phase 3: KNOWLEDGE DIFFUSION AND DisSEMINATION}

Phase 3 starts by expanding the Energy Knowledge Interaction Circles (EKICs) into larger learning groups. The EKICs serve as nuclear or seed groupings holding externalized and formalized energy management knowledge.

Members of the EKICs are intra-organizational energy management experts or super-users.

By having them interact formally (and informally) with larger employee groupings, energy management knowledge is diffused across the organization. The EKIC - employee interaction generates a group-learning scenario, serving to 
raise awareness of energy management issues and practices.

Over an extended period of time organizations observe energy behavioural influences with attendant modulation of long-term energy management behaviour. The energy knowledge dissemination phase is a critical determinant of the success of the KMSE, and it differentiates the approach very clearly from standard energy management schemes. It serves as an important precursive phase to the final KMSE development process.

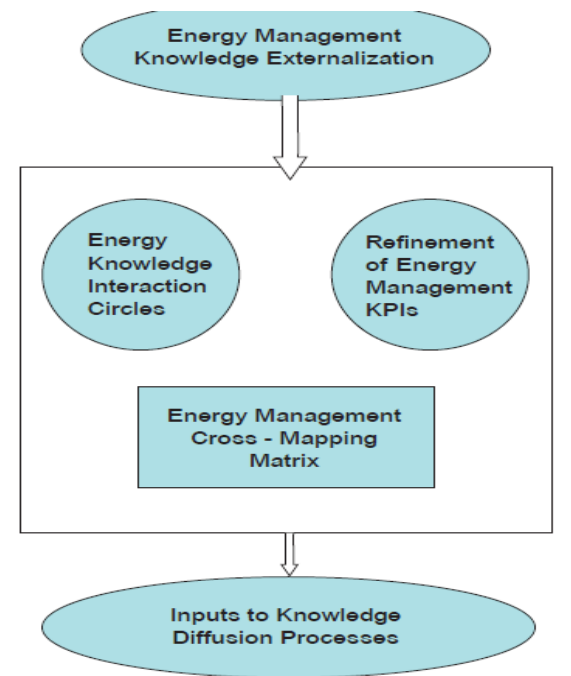

Fig. 4. Energy knowledge externalization phase activities.

\section{Phase 4: KNOWLEDGE INTERNALIZATION AND EXECUTION}

Our discussion of Phase 3 suggested that energy knowledge diffusion would, over a period, start to modulate energy management behavior with the organization. This is essentially the early phases of energy knowledge internalization.

In Phase 4, all energy stakeholders within the organization will have started to internalize more optimal energy management processes and best practices [20]. Additionally, at this point, the organization embarks on a full-scale execution of its designed or redesigned energy management program(s).

The Energy Management program is run with all of its "Monitors" in place. Monitors being specific parts of the KMSE scheme that are devoted to collating energy management efficiency data, for the purposes of composing relevant metrics or KPI items. The execution phase establishes a loop-back for continuous improvement. Energy managers carry necessary corrective or optimization procedures for the KMSE. The loop-back working in conjunction with tracking of performance metrics provides a Quality Assurance mechanism for the KMSE scheme.

It should be re-emphasized that the processes discussed in Phases $1-4$ are fully scalable and may be applied reasonably uniformly to both large-scale and smaller-scale units.

\section{KMSE PILOT STUDY METHODOLOGY AND RESULTS}

To evaluate the efficacy of the KMSE scheme at unit level, and its potential for large-scale sustainable energy management use, a pilot study was carried out with a large Education and Vocational Training Provider in London, UK (hereinafter referred to as the H-Institute). The pilot study carried out data collection and analysis of results, which was typically rendered as Kiviat plots

Hard Kiviat Elements - Based on energy systems analysis (Hard KMSE). The Hard Kiviat plot was cast using the template shown in Fig. 5.

\section{KIVIAT CHART TEMPLATE}

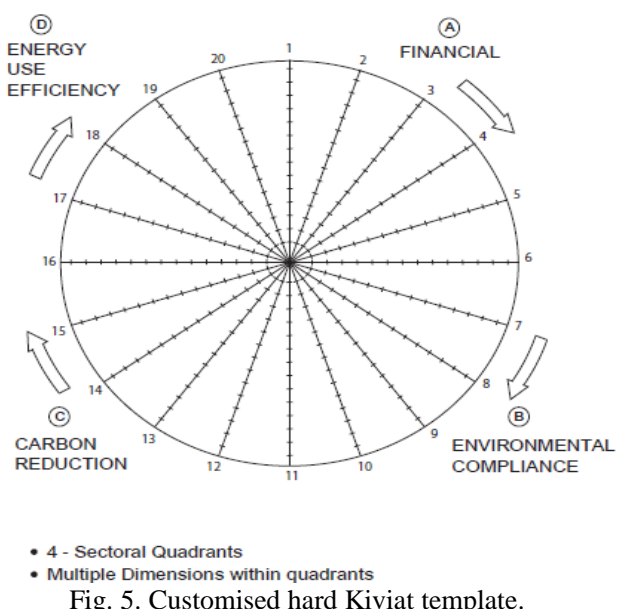

Fig. 5. Customised hard Kiviat template.

\section{HARD ENERGY KIVIAT FORMS}

The prime items within the Kiviat Chart template, when utilized as the 'Hard' Energy Kiviat include:

\section{A. Perspective - Financial}

Sample Dimensions (depicted on normalized scales)

1) Standard Annual Energy cost savings - traced directly or indirectly to energy management program.

2) Initial infrastructural investment on Energy Management infrastructure and program creation

3) Payback period - period for net present value of energy-related cash-flows to switch from negative to positive value.

4) Contribution to Cashflow - from energy program.

5) Return on Energy Investment (ROEI) - metric calculated as (Net Savings)/Total Investment) $\mathrm{x} 100 \%$, over a defined time period.

\section{B. Perspective - Environmental Compliance}

Sample Dimensions:

1) Air Pollution/Air Quality indices - associated with energy generation processes and purchased energy e.g. NOX SOX and particulate emissions attributable to the organisation.

2) Solid waste management: stabilization and disposal from energy generation.

3) Soil and ground water remediation (for sustainability) due to energy generation processes.

4) Hazardous waste management and disposal.

5) Safety indices - related to energy program for operators and general workforce.

\section{Perspective-Carbon Reduction}

Sample Dimensions 
1) Percentage annual reduction in carbon emissions.

2) Estimated absolute tonnage of $\mathrm{CO}_{2}$ emissions from its activities.

3) CRC (Carbon Reduction Commitment) Early Action Metric (EAM) - The percentage of the organizations electricity and other energy supplies covered by voluntary automatic meter readings (AMR) coupled with proportion of CRC Emissions Certified under the 'Carbon Trust Standard' or a similar scheme.

4) CRC Absolute Metric (AM) - Percentage charge in emissions divided by the average emissions for the last five years.

5) CRC Growth Metric (GM) - Percentage in emissions per unit of turnover, divided by the average emissions for the last five years.

Note - This perspective is linked also to the use of alternative (non-fossil Fuel) energy sources.

\section{Perspective - Energy Use Efficiency}

Sample Dimensions:

1) Absolute Energy Consumption in Joules (MJ or GJ)

2) Energy Conversion Efficiency (total facility Power Usage)/Total Power delivered) x $100 \%$, where the total facility power usage is dependent on the aggregated energy consumption ratings of appliances and other energy - consuming devices. This also reflects energy use losses.

3) Energy use variability (maximum Power Usage) (Minimum Power Usage)/Total Power Delivered, where maximum and minimum power usage depend on the premises load.

4) Green Energy Coefficient is the Energy consumed that comes from green sources (Green Energy) divided by total energy consumed by the organization.

5) Energy Reuse Factor is the amount of energy that is sent for reuse (termed 'reuse energy') divided by the total energy consumed by the organization.

\section{Soft Kiviat ElEMENTS - BASED ON ENERGY KNOWLEDGE MANAGEMENT (SOFT KMSE)}

Soft Energy Kiviat forms:

The prime items within the Kiviat Chart template, when utilized as the 'Soft' Energy Kiviat include:

\section{A. Perspective-Awareness and Transparency}

Sample Dimensions (To be depicted on normalized scales):

1) Awareness of energy choices

2) Awareness of energy metrics

3) Awareness of energy policies and strategies

4) Validation of energy schemes

5) Pereception of quality of energy management

\section{B. Perspective - Energy-Related Process Attributes}

Sample Dimensions:

1) Perceived efficiency of energy management processes

2) Internal energy audits

3) Alignment with standards (e.g. ISO 50001/BS EN)

4) Efficacy of corrective processes

5) Effectiveness of maintenance procedures

\section{Perspective - Organizational Factors}

Sample Dimensions:

1) Senior Management commitment

2) Employee Involvement

3) Level of energy-related training

4) Use of qualified "energy" personnel

5) Willingness to fund energy management schemes (e.g. as $\%$ of annual budget)

D. Perspective - Energy-Related Innovation and Learning Sample Dimensions:

1) Range of energy solutions used

2) Degree of customization of solutions

3) Level of documentation of best practices

4) Positivity of energy culture and behaviours

5) Degree of target setting and use of performance monitoring reports

\section{KMSE PILOT STUDY OUTPUT}

The pilot study was carried out over 18 months as an essentially longitudinal project, with cross-sectional data collection elements. Data collection for the "hard Kiviat elements" utilized a mix of primary and secondary energy-usage data.

The primary data for "soft Kiviat elements" was collected through the use of self-completed questionnaires (SCQs) and Semi-Structured Interviews (SSIs) of senior energy decision-makers and energy stakeholders.

The pilot study was carried out over 18 months as an essentially longitudinal project, with cross-sectional data collection elements. Data collection for the "hard Kiviat elements" utilized a mix of primary and secondary energy-usage data. The primary data for "soft Kiviat elements" was collected through the use of self-completed questionnaires (SCQs) and semi-structured interviews (SSIs) of senior energy decision-makers and energy stakeholders.

Using the templates and associated dimensions described earlier, "Hard" and "Soft" Kiviat Graphs were generated for the metric dimensions previously specified.

The figures obtained show "before-KMSE" and "after-KMSE" Kiviat charts, for both hard and soft forms, in Fig. 6-Fig. 9. Larger enclosed areas within the chart are indicative of greater energy management effectiveness.

\section{BEFORE KMSE HARD KIVIAT}

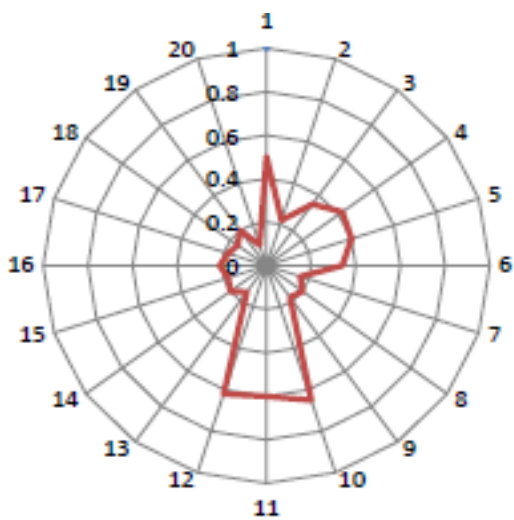

Fig. 6. Pre-KMSE hard Kiviat output plot. 


\section{AFTER KMSE HARD KIVIAT}

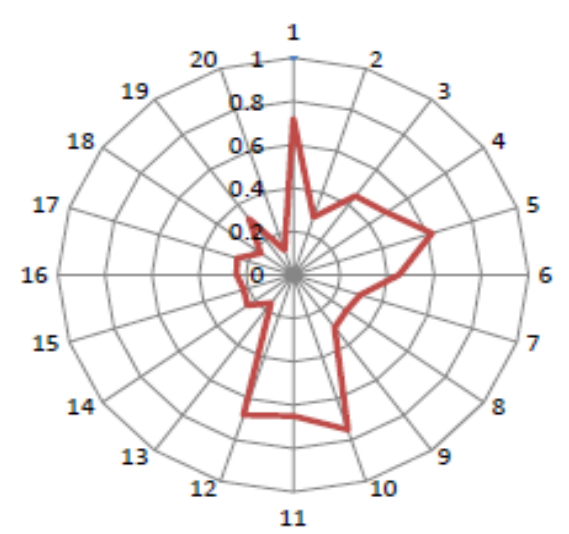

Fig. 7. Post-KMSE hard Kiviat output plot.

\section{BEFORE KMSE SOFT KIVIAT}

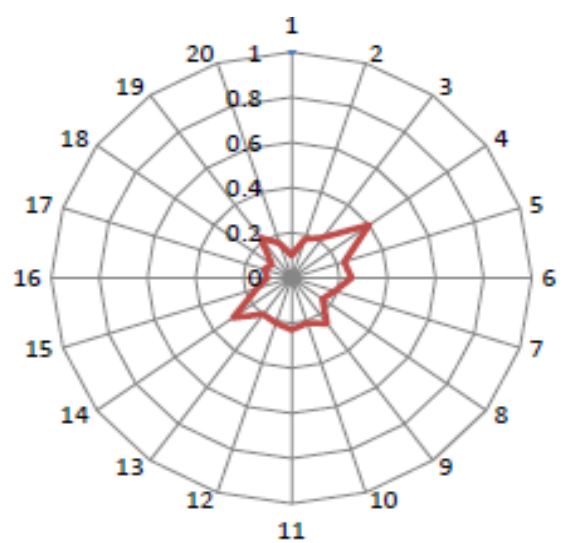

Fig. 8. Pre-KMSE soft Kiviat output plot.

\section{AFTER KMSE SOFT KIVIAT}

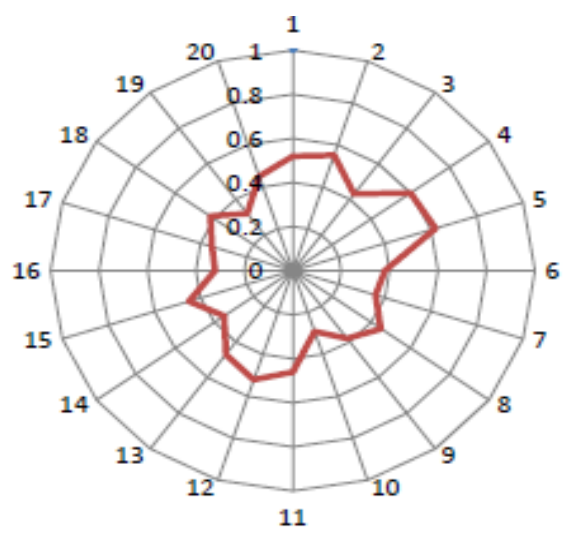

Fig. 9. Post-KMSE soft Kiviat output plot.

\section{KMSE PILOT STUDY CONCLUSIONS}

We observe more marked changes for the Soft Kiviat, across nearly all dimensions.

There were also some significant changes for the Hard Kiviat, particularly for dimensions within the financial, environmental and energy use efficiency quadrants.

The Hard KMSE dimensions are generally lagging indicators - benefits take longer to feed through to these items.

Overall the pilot study results suggest that there could be potential benefits from using such a scheme, particularly when scaled up.

\section{ON-GOING ENERGY RESEARCH WORK AT RAK}

To underpin a KMSE scheme, it is beneficial to provide enhancements in available energy technologies, and widen the choices available. Also energy solutions need to be contextualized to the local environment.

Our past and current (ongoing) energy-related research at RAK has been in the following areas:

1) Photovoltaic (PV) solar lighting schemes

2) Parabolic trough concentrated solar power schemes

3) Wave energy power generators - with unique float mechanism design

4) Grey water energy harvesting schemes

5) Novel energy \& pollution monitoring systems

\section{ACKNOWLEDGMENT}

The author gratefully acknowledges the support provided by the American University of Ras Al Khaimah, and is appreciative of the educational consultancy input provided by the consultancy teams at Eden Management Consultants (Educational Unit) and Hanta Associates, as well as valuable discussions with relevant academic Faculty of the University of Bolton, Bolton-UK and Ras Al Khaimah-UAE Campuses.

\section{REFERENCES}

[1] A. Bergek, S. Jacobsson, B. Carlsson, S. Lindmark, and A. Rickne "Analysing the functional dynamics of technological innovation systems: A scheme of analysis," Research Policy, vol. 37, pp. 407-427, 2008.

[2] S. A. Carlsson, "Knowledge managing and knowledge management systems in inter-organisational networks," Knowledge and Process Management, vol. 10, no. 3, pp. 194-206, 2003.

[3] G. C. Gallopín, "Environmental and Sustainability Indicators and the Concept of Situational Indicators. A Systems Approach," Environmental Modelling \& Assessment, vol. 1, no. 3, pp. 101-117, 1996.

[4] V. Grover and T. H. Davenport, "General perspectives on knowledge management: Fostering a research agenda," Journal of Management Information Systems, vol. 18, no. 1, pp. 5-21, 2001.

[5] J. Houghton and P. Sheehan, "A primer on the knowledge economy," Notes, vol. 18, no. 18, p. 25, 2000.

[6] G. P. Huber, "Organisational learning: The contributing processes and the literature," Organisation Science, vol. 2, no. 1, pp. 88-115, 1991.

[7] S. Jacobsson and A. Bergek, "Energy system transformation: the evolution of technological systems in renewable energy technology," Industrial and Corporate Change, vol. 13, no. 5, pp. 815-849, 2004.

[8] D. Kelleher and S. Levene, Knowledge Management: A Guide to Good Practice, British Standards Institute: London, 2001.

[9] T. Knight and T. Howes, Knowledge Management: A Blueprint for Delivery, Butterworth Heinemann: Oxford, 2003.

[10] P. J. Lane and M. Lubatkin, "Relative absorptive capacity and interorganisational learning," Strategic Management Journal, vol. 19, no. 5, pp. 461-477, 1998.

[11] S. Newell, M. Robertson, H. Scarbrough, and J. Swan, Managing Knowledge Work and Innovation, London: Palgrave Macmillan, 2009

[12] OECDb, "Conclusions from the results of the survey of knowledge management practices for ministries/departments/agencies of central government in OECD member countries," GOV/PUMA/HRM, 2003.

[13] J. C. Pai, "An empirical study of the relationship between knowledge sharing and IS/IT strategic planning (ISSP)," Management Decision, vol. 44, no. 1, pp. 105-122, 2006.

[14] P. Trusts, "Who is winning the Clean Energy Race?" The G-20 Clean Energy Fact Book, 2010.

[15] H. Scarbrough, Knowledge Management: A Literature Review, Issues in People Management Series, Institute of Personnel and Development: London, 1999. 
[16] M. Soekijad et al., "Learning and knowledge processes in inter-organisational communities of practice," Knowledge and Process Management, vol. 11, no. 1, p. 312, 2004.

[17] M. S. Sohail and S. Daud, "Knowledge sharing in higher education institutions," VINE: The Journal of Information and Knowledge Management Systems, vol. 39, no. 2, pp. 125-142, 2009.

[18] R. K. Turner, "Sustainability: principles and practice," in Sustainable Environmental Economics and Management: Principles and Practice, R. K. Turner Ed. Belhaven Press, London. pp. 3-36, 1993.

[19] E. Wenger, R. McDermott, and W. M. Snyder, "Cultivating communities of practice," Management Science, vol. 54, p. 284, 2002.

[20] L. Xia and S. Ya, "Study on knowledge sharing behaviour engineering," Systems Engineering Proceedings, vol. 4, pp. 468 - 476 , 2012.

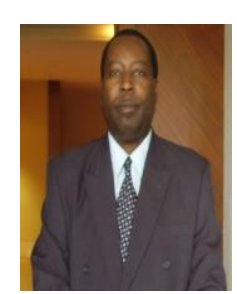

Anthony Ayoola has a multi-disciplinary background in business management, computing and engineering, with degrees from Oxford and London Universities (BA, MA, MSc and $\mathrm{PhD}$ degrees). He is currently the dean of the Business School at the American University of Ras Al Khaimah.

He has been lecturing, carrying out research and engaging in consultancy for over 30 years, and has worked in the United Kingdom, United States, Netherlands, Africa and the Middle East. He has authored several external and internal publications over this period. 